\title{
Cross-layer Optimization in the Next-generation Broadband Satellite Systems
}

\author{
I. Melhus ${ }^{1}$, L. Fan $^{2}$, H. Cruickshank ${ }^{2}$, F. Arnal ${ }^{3}$, C. Baudoin ${ }^{3}$, F. Nivor ${ }^{4}$, T. Gayraud ${ }^{4}$, \\ F. Rodriguez ${ }^{5}$, A. Pietrabissa ${ }^{6}$, L. Lei ${ }^{2}$, Z. Sun $^{2}$ \\ ${ }^{1}$ SINTEF, Norway \\ ${ }^{2}$ University of Surrey, UK \\ ${ }^{3}$ Thales Alenia Space-France \\ ${ }^{4}$ LAAS-CNRS, France \\ ${ }^{5}$ Telespazio SpA, Italy \\ ${ }^{6}$ University of Rome, Italy
}

\begin{abstract}
Next-generation broadband satellite systems will have the capability to provide costeffective universal broadband access for the users. In order to meet users' requirements on high quality multimedia services, many enhancements have to be made on the existing satellite technologies. One of the promising methods is the introduction of cross-layer design. There are several advantages of a layered approach since modularity, robustness and ease of designs are achieved without difficulty. However the properties of the different layers have substantial interdependencies and a modularised design may therefore be suboptimal with regards to performance and availability in a hybrid satellite and mobile wireless environment. In this paper, we will carry out a review of the cross-layer design in satellite systems. Based on this, a cross-layer architecture for the next-generation broadband satellite system is proposed. The proposed cross-layer architecture has two main components: QoS and resource management and mobility management. In each component, the cross-layer techniques that have been used are described in details.
\end{abstract}

\section{Introduction}

Cross-layer architectures diverge from the existent network design approaches, where each layer of the protocol stack operates independently and the data between the successive layers is exchanged in a very strict and systematic manner. There are several advantages of a layered approach since modularity, robustness and ease of design are easily achieved. In view of the fact that a layer has to perform certain functions, the design efforts only need to be focused on these functions without concerning the properties and interactions with other layers. The modularity that the layers provide allows for potential arbitrary combination of protocols, and the maintainability is being improved as new versions of a protocol can be inserted without having to alter the rest of the network stack. However the properties of the different layers have substantial interdependencies and a modularised design may be suboptimal with regards to performance especially in hybrid satellite and mobile wireless environments, where the communication channels and traffic patterns are more unpredictable than in wired-line networks.

In case of cross-layering, information is allowed to be exchanged between adjacent and non-adjacent layers of the protocol-stack, by means of a broader and much more 
open data format. In this way the overall system performance can be improved by taking advantage of the available information across different layers. Cross-layer processing adapts the link-, network- and transport-layer parameters to the channel and the applications instantaneous requirements. To achieve these optimization goals, appropriate signalling methods and architectures for cross-layer designs are necessary. Two alternative architectures for cross-layer design are summarized below:

- Direct communications architecture where uni- or bi-directional transfer of information between two adjacent or nonadjacent layers are implemented. This is done by creating new interfaces at the selected layers beyond those normally used between the layers.

- Indirect communications architecture where instead of doing communications between specific layers, the cross-layer design utilize a parallel structure that acts as a shared database of the system state, being accessible to whichever layers who choose to utilize it.

This paper describes cross-layer design architectures and interaction techniques as implemented and proposed in the EU-funded IST FP6 project Satellite-based communication systems within IPv6 (SATSIX). This includes cross-layer techniques for both direct and indirect communications between layers. Based on existing solutions a cross-layer interaction module (CLIM) adapted to satellite network has been proposed. The main areas for cross-layer design have been identified to be related to QoS and Radio Resource Management and Mobility Management.

The QoS and Resource Management component includes:

- $\quad$ SIP and MAC cross-layer interactions are used to support the interworking between WiMAX and DVB-RCS, and multimedia QoS-aware application.

- Transport layer and MAC cross-layer interaction (i.e. the interaction between TCP PEP and DAMA in the MAC) is designed to optimise the way in which the available resource is used taking into account QoS mapping at the MAC layer and enables data to be sent to the lower layers at the speed at which the MAC layer queues are emptied (flow-control).

- IP and MAC scheduling interaction are implemented in a way that can fully take advantage of QoS capabilities offered by the satellite system.

- MAC and Physical layer interaction between DRA and DAMA as information in the frame constitution.

Details of the CLIM architecture adapted to these areas are described and examples shown how to implement this model and other equivalent interaction models.

\section{Cross-layer feedback issues}

The solutions for cross-layer adaptation seek to enhance the performance of the system by jointly designing the performance of single or multiple cross-layers. The question is to what extent the layered architecture needs to be modified in order to introduce co-operations among protocols belonging to different layers. At one end, solutions based on layer triggers implement interdependencies between protocols maintaining compatibility with strict layering. A full cross-layer design represents the other extreme; introducing stackwide layers' interdependencies that enable the optimization of each protocol's performance by exploiting the full knowledge of the 
network status collected at different layers of the protocol stack:, the status of the wireless links connecting a node to its neighbours, the network topology.

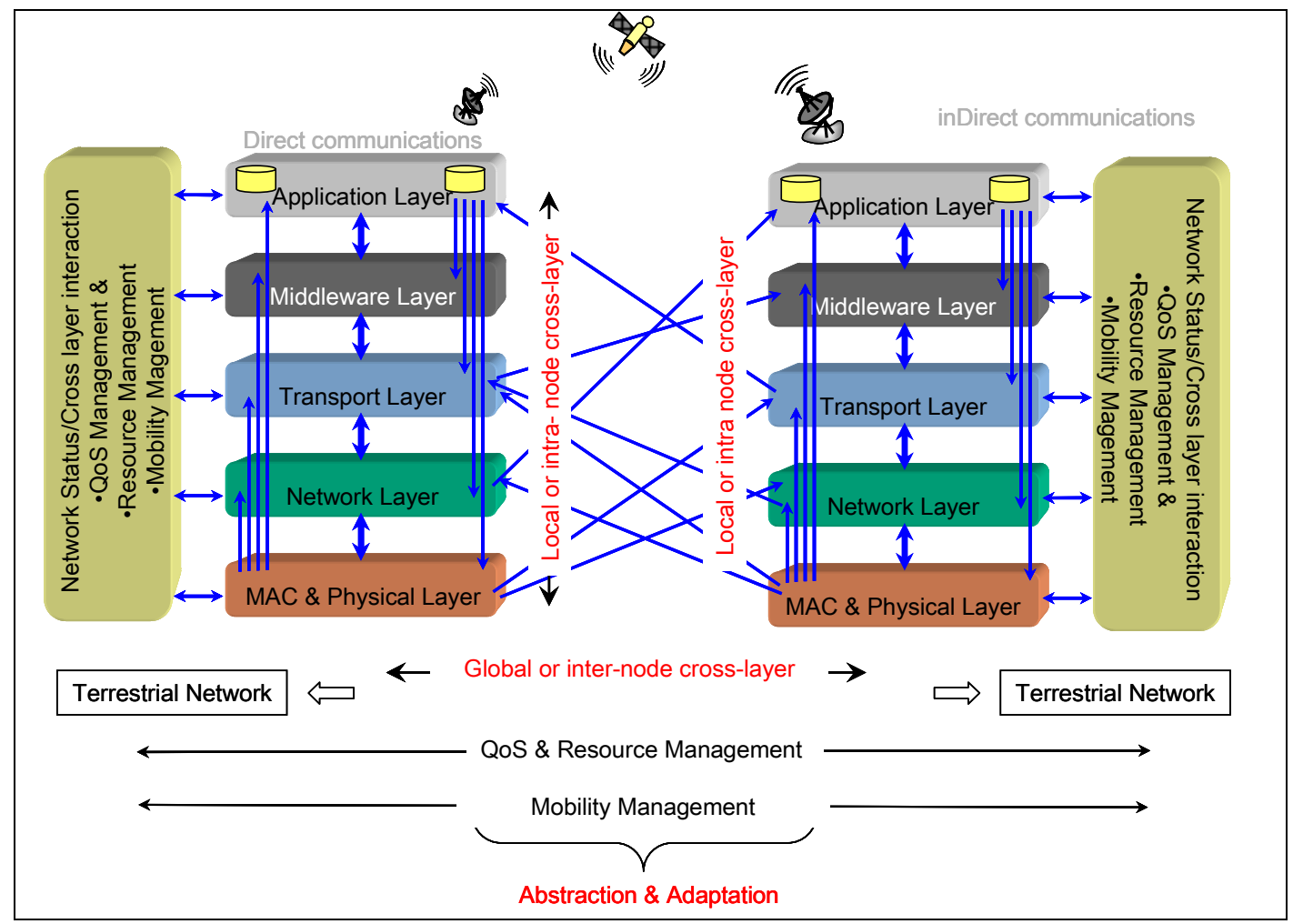

Figure 1 Cross-layer adaptation and optimization in satellite network

Figure 1 illustrates the main ideas of cross-layer adaptation and optimization in a hybrid terrestrial and satellite network. Cross-layer feedback optimisation may be implemented locally (intra-node) or globally (inter-node), both at the intermediate nodes or at the mobile end hosts. However, in a multiple-objective optimization scenario, care should also be taken to avoid undesirable (and unpredictable) interactions across parameters in various layers, leading to conflicts or loops between the layers. There will also be design trade-offs between the multiple optimization goals and the effect of the increased processing and interactions to achieve these goals.

A number of cross-layer methods and architectures have been proposed in the literature [srivastava05] [giambene06] [wang] [winter] [eclair]. They all share some common features and diverge notably in the way the cross-layer principle is implemented, what kind of application focus, the capacity the architecture has and where the actual adaptation intelligence is located. These architectures mostly fit out into one of the two categories: direct cross-layer communications and indirect crosslayer communications via a common entity, see Figure 2. The first category direct communications should be used when only a single cross layer optimization is planned. The second category indirect communications is realised with a common cross-layer entity or cross-layer manager, which acts as a mediator between the layers. The cross-layer entity includes a network status component of the stack that interfaces the different layers between themselves, and it acts as a database where each network layer can put or get information. This architecture should be used for multiple cross layer optimizations. 


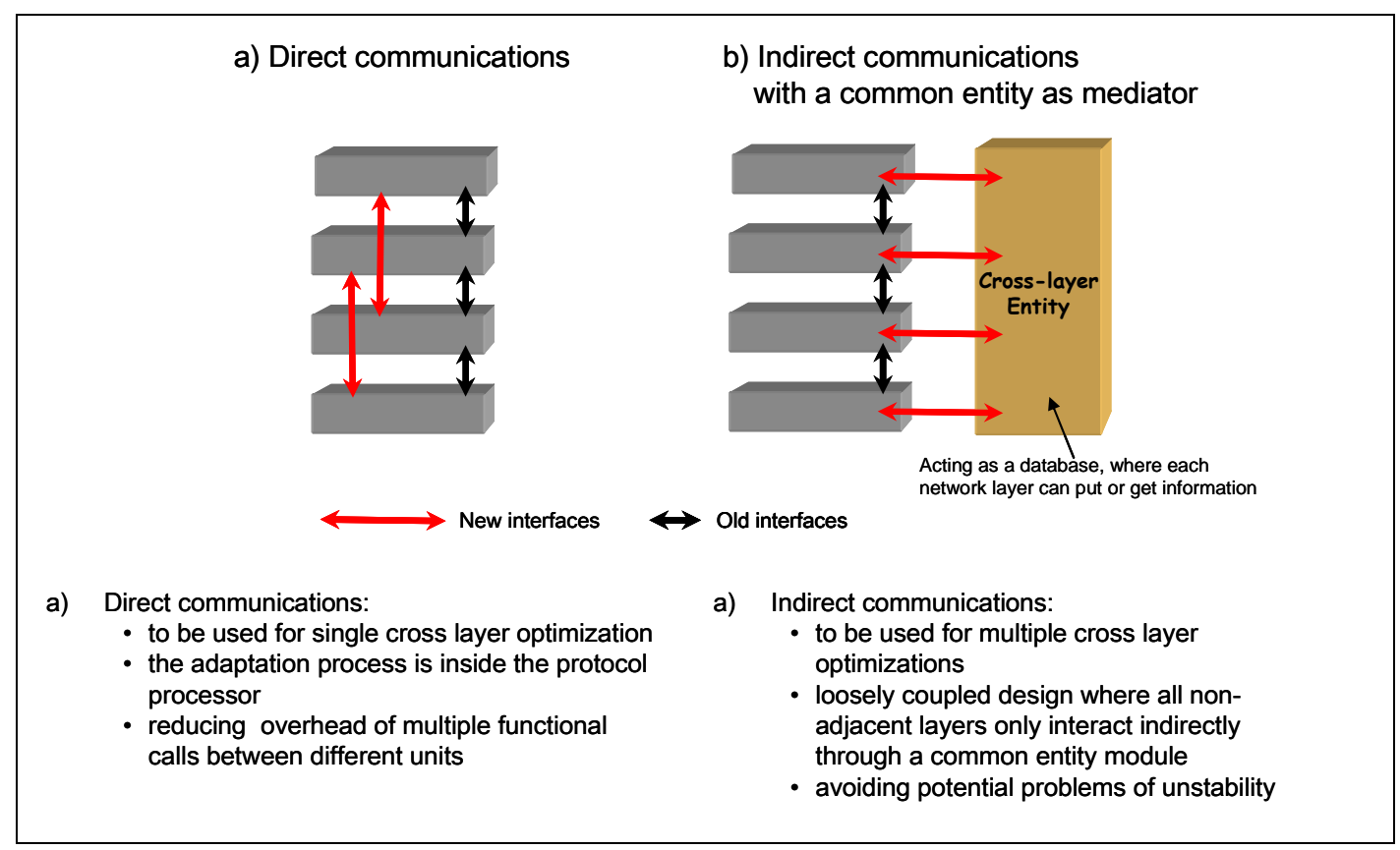

Figure 2 Cross-layer architectures for indirect and direct communications

In satellite and wireless network it is expedient to do adaptation and interaction that violates the networks traditional layered architecture. Unfortunately by doing so in an undisciplined way it is likely to end with a poorly structured system and to greatly increase the complexity of an already complex system [choi] [raisinghani].

\section{New Cross Layer Interaction Model (CLIM)}

The cross-layer architectures proposed in the literature that focus on cross-layer interaction within the protocol stack do not address all the design goals of interoperability, rapid prototyping, maintainability, portability, and efficiency. Starting from this consideration, our goal is to provide a generic framework for building and organising a cross-layer interactions model (CLIM) which could serve as a unified and simple way to "implement" CL optimisations, see Figure 3. The concept of Network Feature (NF) is also introduced. A NF is either a functional service that can be provided to the end-user (e.g. QoS), or a network component whose operations/configurations are supposed to be critical in terms of performance, efficiency or services, at the system level or for the user satisfaction. Basically, crosslayer interactions may be local to or distant within a network node. In many cases only two elementary Network Features (NF) might be involved in the adaptation and interaction (one source NF, one target NF). In some other cases, however, multiple (local or distant) entities could also participate. Local communications between protocols of non-neighbour layers are done through a local interface that must be created for all layers involved in cross-layer interactions.

The proposed cross-layer architecture and interaction module has two main components: QoS and Resource Management and Mobility Management. In each component, the cross-layer techniques that have to be used are defined. 


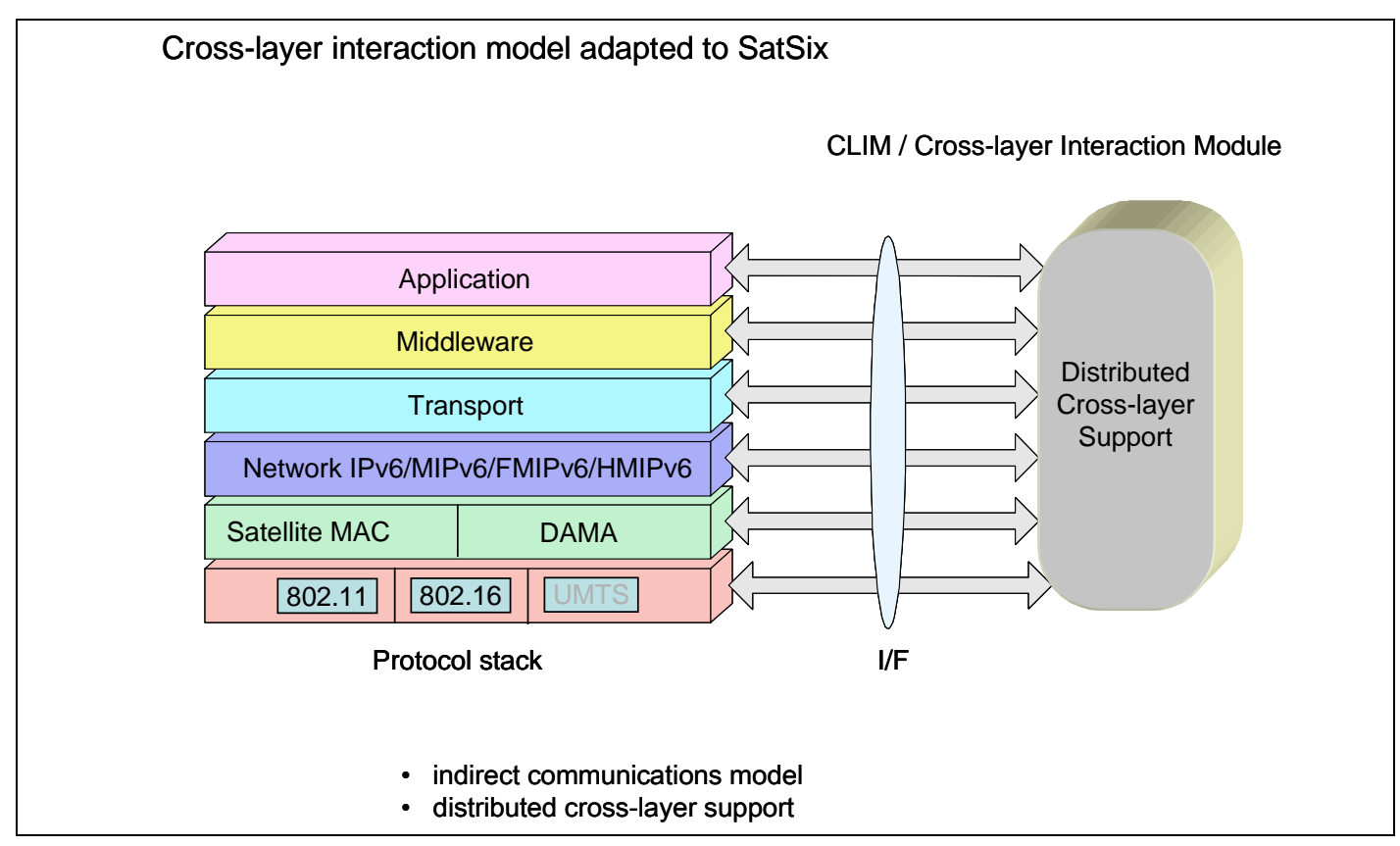

Figure 3 Proposed Cross-Layer Interaction Model

The Mobility Management component utilizes a revised model (CLIM-m) based on the more generic CLIM architecture and ideas from [biya] [mohanty], see Figure 4. The cross-layer mobility management modules include:

- Algorithms for handover prediction and decision algorithms for fast handover with handover preparation, handover coordination and optimization algorithms for best performance.

- Information to decide the appropriate time to initiate and execute the handover procedures.

- Functionalities or algorithms for a particular policy based cross layer optimization or tuning could also be included. 


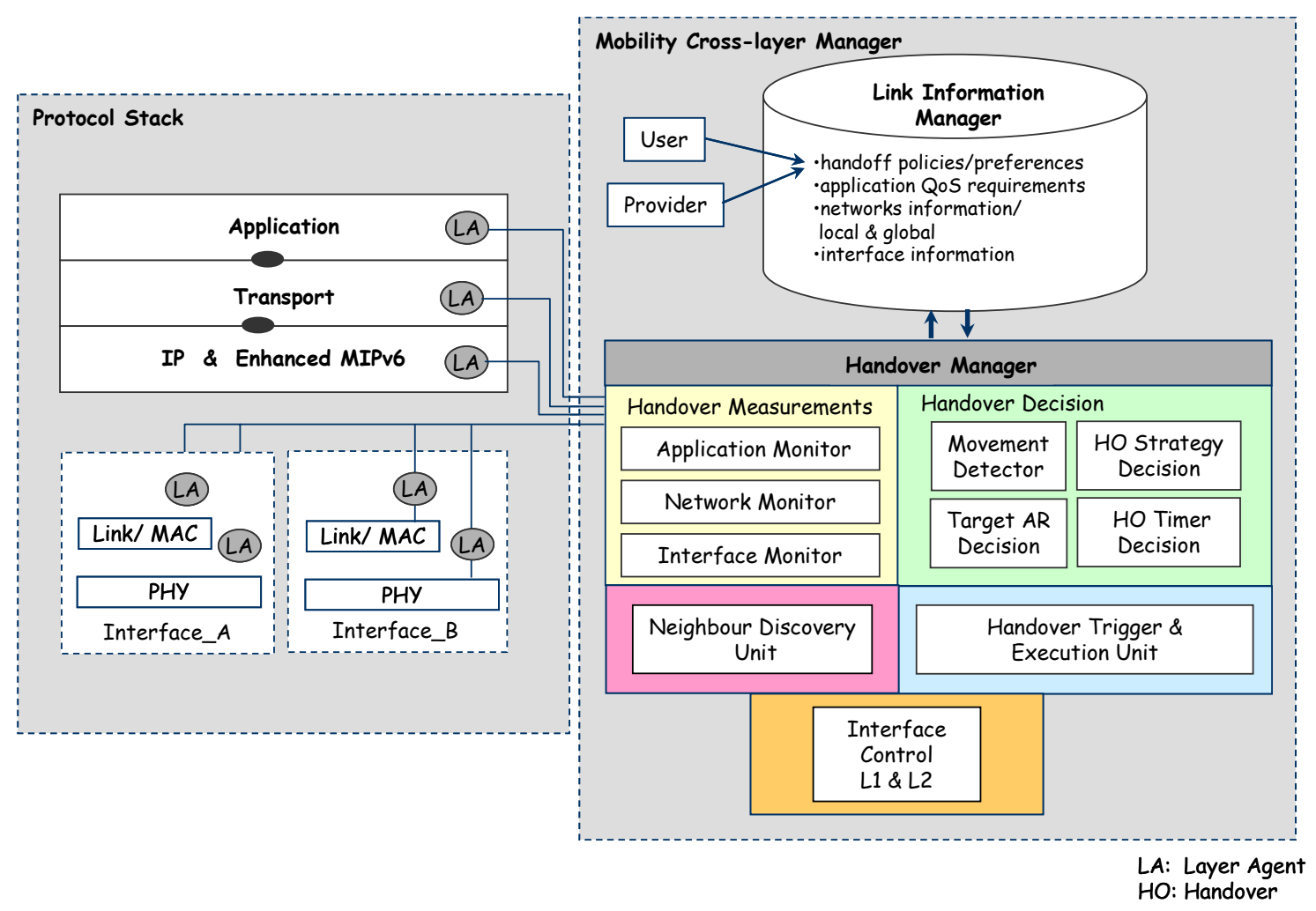

Figure 4 Mobility cross-layer architecture (CLIM-m)

The proposed cross-layer platform specifies a Cross-layer Manager consisting of a Link Information Manager and a Handover Manager connected to Layer Agents (LA). The Handover Manager communicates with multiple protocol layers via the Layer Agents which will capture specified parameters in each layer when the respective values are changing beyond a certain threshold. These data are reported to the Handover Manager to collect sufficient information for the Handover Decision unit.

\section{Details of cross-layer implementations in SATSIX networks}

\section{A. Approaches managed by CLIM architecture}

Since most of the interactions that has been tuned or derived for SATSIX inherit from previous implementations, only direct exchanges between dedicated NFs with customised interfaces are actually implemented, without making use of the Cross Layer Manager Entities. However Figure 4 shows how direct interactions could be addressed in a CLIM-based approach. 


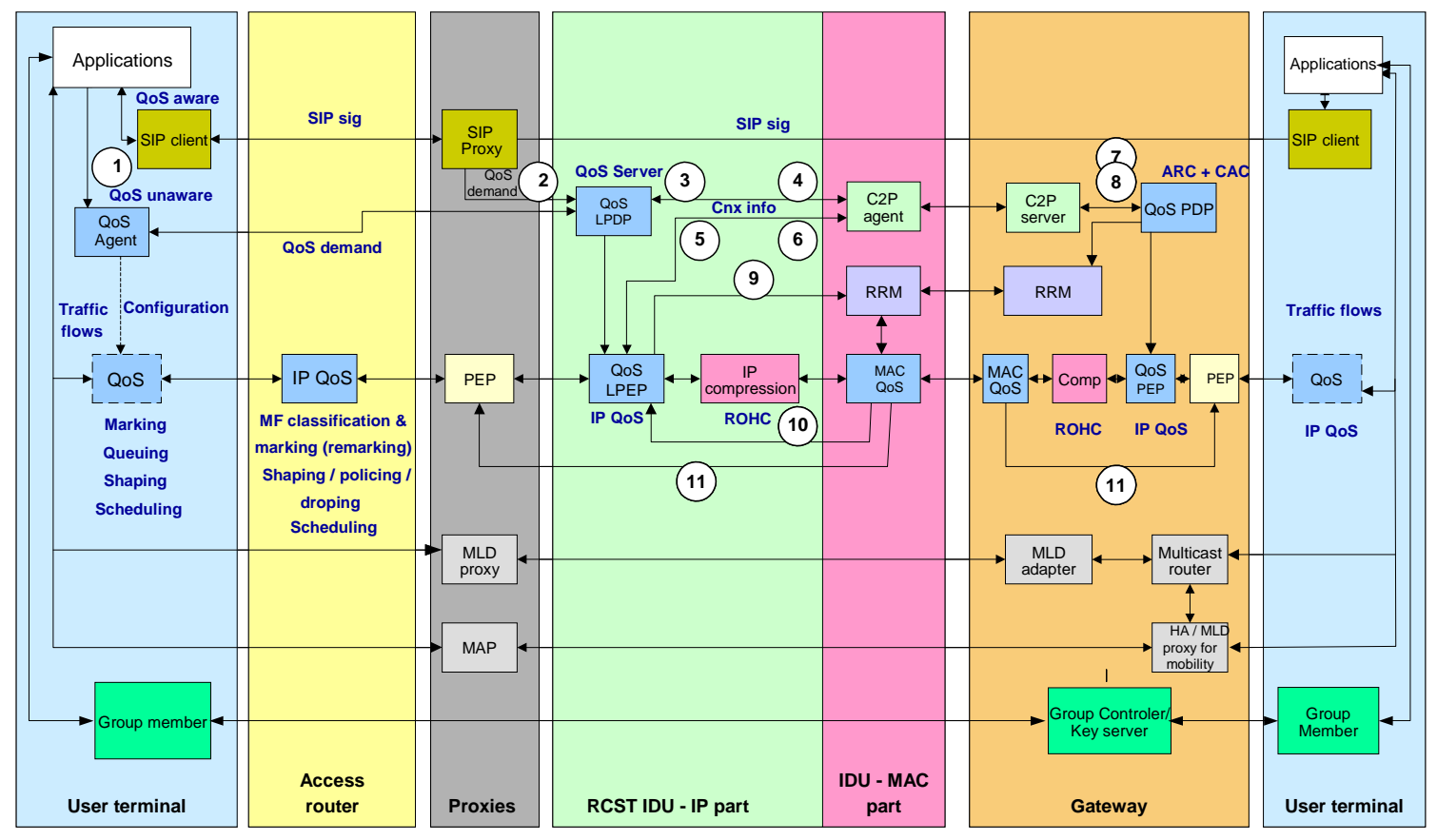

Figure 5 Identified cross-layer interactions in SATSIX functional network architecture

$I_{1}$ : Application $\rightarrow$ QoS Agent :The QoS Agent is meant to detect traffic flows to be processed and to configure local and remote QoS mechanisms according to the traffic flows characteristics (essentially addresses and port numbers).

$I_{2}:$ SIP Proxy $\rightarrow$ QoS LPDP (QoS Server): In this interaction, the SIP proxy extracts and maps the incoming session description to a set of QoS parameters that will be used at the QoS server to provision MAC resources (for example, through the C2P protocol).

$I_{3}:$ QoS LPDP (QoS Server) $\rightarrow$ C2P Agent: In the SatSix MAC-oriented network architecture, MAC connections at the satellite system level may be created, modified or deleted according to the C2P signalling procedures. In this interaction, the QoS server expresses a QoS requirement for a new traffic flow to the C2P Agent which will possible decide the mapping to be done between IP flows and MAC flow identifiers.

$I_{4}$ C2P Agent $\rightarrow$ QoS LPDP: The purpose of this interaction is to inform the local QoS server of the result of the previous request, based on the CAC output communicated to the $\mathrm{C} 2 \mathrm{P}$ server (and sent to the $\mathrm{C} 2 \mathrm{P}$ agent).

$I_{5}$ C2P Agent $\rightarrow$ QoS LPEP: Upon a new C2P connection request (see $\mathrm{I}_{6}$ ), the C2P agent provides the IP scheduler the granted QoS for this flow.

$I_{6}$ QoS LPEP $\rightarrow$ C2P Agent: For QoS unaware applications, a new incoming IP flow at the IP scheduler would have to trigger a request for opening a new C2P connection with a given QoS. 
$I_{7}$ C2P Server $\rightarrow$ QoS PDP (CAC): In this interaction, the admission control is performed for a new connection; it may either result on the creation of a new MAC connection, or to the modification of an existent one.

$I_{8}$ QoS PDP $(C A C) \rightarrow C 2 P$ Server: In response to the previous request, the CAC indicates to the C2P server if the new connection may be accepted or not.

$I_{9}$ QoS LPEP $\rightarrow$ RRM: As well as MAC buffers are measured to provide inputs for the RRM, IP queues could be measured in order to anticipate allocation resources requests.

$I_{10}:$ MAC QoS $\rightarrow$ QoS LPEP: In this interaction (relevant for mixed IP/MAC QoS architecture with back pressure congestion control), the output data rate at the IP scheduling is adjusted according statistics of MAC buffers.

$I_{11}$ MAC QoS $\rightarrow$ Transport PEP: This interaction purpose is to coordinate output data rate from the PEP buffer with the MAC instantaneous available rate.

\section{B. Approaches not specifically managed by CLIM architecture}

The main concern in the satellite communication context, and more particularly on satellite return link, is to make an efficient use of the resources, scarce and costly, while taking into account different traffic types (data, voice, video), each having specific QoS performance parameters. Thus, protocols have been designed to optimize the use of these resources and especially to share properly and efficiently the return link resources accessed by multiple distributed Satellite Terminals (ST) which acts as satellite boundary nodes. In the satellite networking context, the interaction between the application-layer, where the QoS might be set, and the lower layers where the traffic is finally prioritized is not covered by any standard specification. For example, the physical layer QoS, such as Adaptive Coding \& Modulation (ACM) can be used by higher layers to optimise the radio resource management. The Link-layer QoS, such as scheduling and BoD can deal with both physical and transport-layer to optimise the system performance.

QoS support for multimedia QoS-aware applications:

The standard protocol published by IETF for setting up multimedia session applications is the Session Initiation Protocol (SIP). Figure 6 shows the functional operation of SIP in a DVB-S/RCS satellite system with integrated DAMA support. 


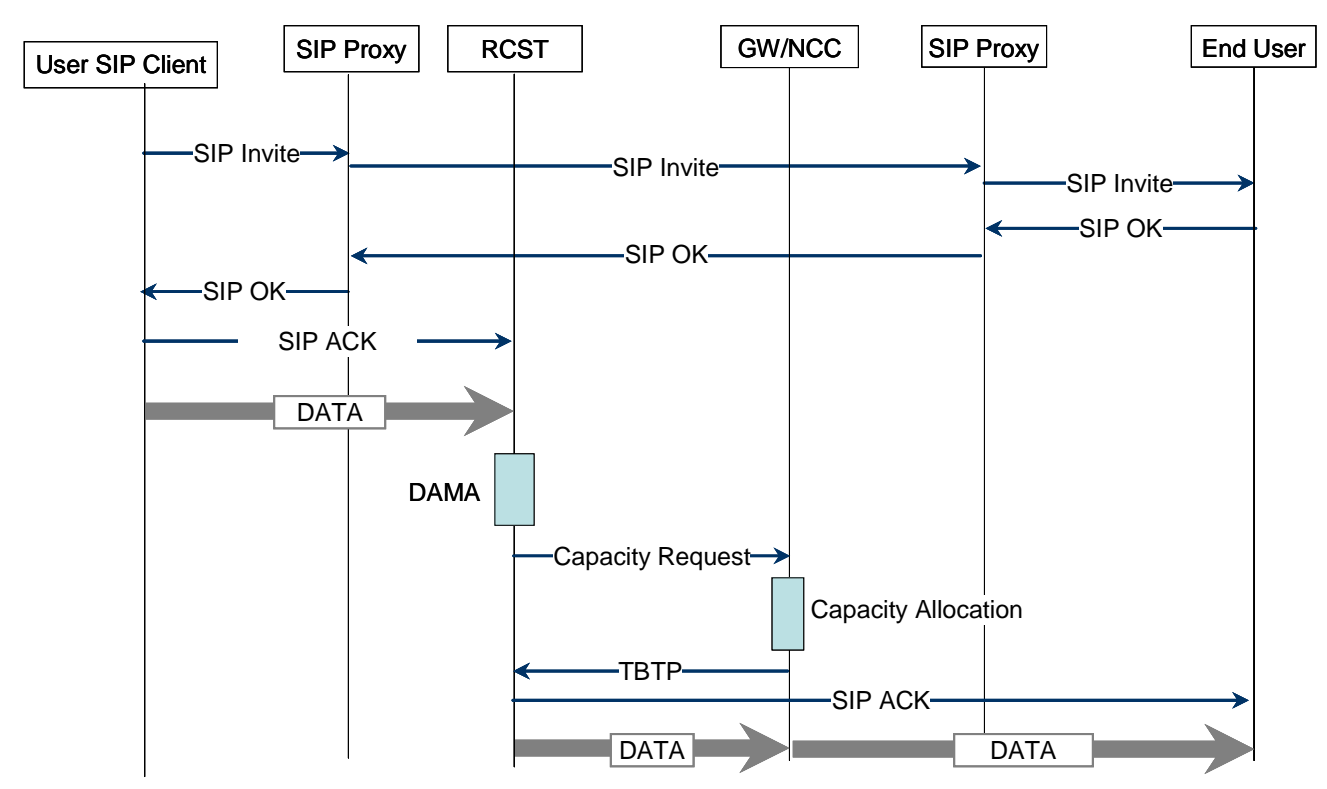

Figure 6 SIP functional operation

- The DAMA is triggered for dynamic bandwidth allocation in the satellite network. This can be performed both at IP and MAC level. In the latter the class of services are mapped onto the DVB-RCS capacity requests (i.e. Continuous Rate Assignment (CRA), Rate Based Dynamic Capacity (RBDC), Volume Based Dynamic Capacity (VBDC), Absolute Volume Based Dynamic Capacity (AVBDC) and Free Capacity Assignment (FCA)).

- The capacity requests are sent to the NCC via SYNC burst.

- CAC (Conditional Access control) will check whether the required capacity is available.

- NCC will, if they are available, allocate resources and send the TBTP to RCST.

It uses a highly efficient assignment type of dynamic access scheme, and concerns the anticipation for provisioning on-demand resources on the communication opening and the improvement of the request resource algorithm (permanent state) in order to turn it more dynamic. It can optimally use of the resources on the return link channel, and a significant decrease of the latency in the satellite terminals.

\section{WiMax and DVB-RCS interworking:}

Both WiFi and WiMAX terrestrial access networks may be connected to the RCSTs to provide shared access to the traffic forwarded on the satellite link. The SIP and MAC cross-layer interactions are now used to support the interworking between WiMAX and DVB-RCS [rodriguez]. 
Every time a new connection is set up the user application sends SIP parameters, which can be intercepted by the RCST and if the WiMAX authorization process has success, a MAC connection is established at WiMAX level and starting then to send packets without a real relation between the two sub networks.

Moreover, if the dynamic bandwidth allocation is allowed at WiMAX node then the application should renegotiate the QoS parameters through SIP protocol and stop the services it is supporting. Actually when the dynamic definition and change of service flows take place, a possible overriding of the provisioned QoS parameters negotiated can exist.

The SS can modify its behaviour and renegotiate the QoS parameters of the active CID, but this renegotiation cannot be intercepted by the satellite subnetwork. So ideally there should be an information exchange between the Authorization Manager and the satellite bandwidth allocation management process (DAMA) for a dynamical redefinition of QoS parameters before allowing any change in the service flow profile.

\section{Efficient resource management scheme:}

The proposed scheme is based on a third part agent (the BoD controller) that collects information from the lower layer (MAC layer) and sends them to the upper layer (IP layer) in order to dynamically enable and disable the scheduler. This mechanism has been developed to improve performances in a congested scenario and allows a better resource exploitation in terms of packet loss and access queuing delay. The key idea of the proposed algorithm is that it forces the scheduler to send to the lower level only the exact amount of packets to fulfil the available bandwidth assignments.

\section{Conclusions}

In cross-layer design the overall system performance can be improved by taking advantage of the available information across different layers. Having additional data to build decisions on and to parameterize and to adapt protocol behaviour can tremendously increase a protocol's performance. This paper has first given a generic approach to cross-layer design and optimization. Based on the existing solutions a cross-layer interaction module (CLIM) adapted to satellite network is proposed. The main areas for cross-layer design in identified for satellite networks are within QoS and Radio Resource Management and Mobility Management. Details of CLIM architecture adapted to these areas are described and examples on how to implement this model and equivalent models are shown. Other approaches not specifically managed by the CLIM architecture re also described.

\section{Acknowledgement}

This work is supported by the IST FP6 SATSIX project, funded by European Commission (EC). The financial contribution of the EC towards this project is greatly appreciated. 


\section{References}

[biya] Yana Bi, Mei Song, Junde Song: "Seamless mobility Using Mobile IPv6”, http://netlab18.cis.nctu.edu.tw/html/InternetProtocol/2005/paper/Seamless\%2 0mobility\%20using\%20mobile\%20IPv6.pdf.

[choi] Soon-Hyeok Choi, Dewayne E. Perry and Scott M. Nettles: "A Software Architecture for Cross-LayerWireless Network Adaptations”, The University of Texas at Austin Austin, Texas 78712 fschoi, perry, nettlesg@ece.utexas.edu

[eclair] V. T. Raisinghani and S. Iyer: "ECLAIR: An efficient cross layer architecture for wireless protocol stacks”, WWC2004, http://www.it.iitb.ac.in/ rvijay/research/phd/papers/wirelesscongress/ eclair_wwc04.pdf

[giambene06] Giovanni Giambene and Sastri Kota: “Cross-layer protocol optimization for satellite communications networks: A survey”, Int. J. Satell. Commun. Network. 2006

[mohanty] Shantidev Mohanty and Ian F. Akyildiz: "A Cross-Layer (Layer $2+3$ ) Handoff Management Protocol for Next-Generation Wireless Systems” IEEE Transactions on Mobile Computing, vol. 5, no. 10, Oct 2006.

[raisinghani] Vijay T. Raisinghani, Sridhar Iyer: "Cross Layer Feedback Architecture for Mobile Device Protocol Stacks”, http://ieeexplore.ieee.org/iel5/35/33395/01580937.pdf

[rodriguez] Filippo Rodriguez et al.: "Interworking Strategy between DVB-RCS and WiMax”, INNSS’07, IST-Summit, Budapest 2007

[srivastava05] Vineet Srivastava and Vineet Srivastava: "Cross-Layer Design: A Survey and the Road Ahead”, IEEE Communications Magazine, December 2005, http://ieeexplore.ieee.org/iel5/10596/33500/01589660.pdf?tp=\&isnumber=\&a rnumber $=1589660$

[tiado] M. Issoufou Tiado, "Modeles et mécanismes multiniveaux pour les réseaux sans fil” Ph.D thesis of Institut National Polytechnique de Toulouse, 2006

[vanderschaar] M. Van der Schaar, D. Sai Shankar, "Cross-Layer Wireless Multimedia Transmission Challenges, Principles, and New Paradigms”, Wireless Comunications, Vol 12, Issue 4, pp 50-58, August 2005

[wang] Qi Wang and Mosa Mi Abu-Rgheff: “A Multi-Layer Mobility Management Architecture Using Cross-Layer Signalling Interactions" http://www.cis.udel.edu/ yackoski/cross/qwang_epmcc03_paper.pdf.

[winter] R. Winter et al., "CrossTalk: A Data Dissemination-Based Crosslayer Architecture or Mobile Ad Hoc Networks,” Proc. ASWN, June 2005. 